\title{
A Simulation Technique for RF Amplifier Circuits Using ANSYS Electronics Desktop
}

\author{
Marcelo P. Magalhães, Marcos V. T. Heckler \\ Universidade Federal do Pampa (UNIPAMPA) \\ Alegrete, Rio Grande do Sul, Brazil \\ marcos.heckler@unipampa.edu.br
}

\author{
Andreas Winterstein, Lukasz A. Greda \\ Institute of Communications and Navigation \\ German Aerospace Center (DLR) \\ Oberpfaffenhofen, D-82234 Wessling, Germany \\ andreas.winterstein@dlr.de
}

\begin{abstract}
This paper describes a simulation technique for RF amplifier circuits using an electromagnetic simulator. To validate the proposed approach two RF circuits were designed, manufactured and measured. Simulated and measured results were compared and good agreement was observed.
\end{abstract}

\section{INTRODUCTION}

For the design of radio frequency (RF) circuits, it is advantageous to assess the expected performance prior to manufacturing. This speeds up the development process and saves resources. Although specialized tools to simulate complete RF systems exist they are very expensive and may not be available to the antenna engineer.

In this paper we propose and validate a simulation technique for PCBs with RF amplifiers using electromagnetic software packages that are normally used for simulations of antennas and other microwave structures. The proposed technique will be explained based on ANSYS Electronic Desktop (AEDT) [1] but similar software packages may be used.

\section{PCB Simulation Methodology}

The simulation technique consists basically of three steps. In the first step the PCB layout is designed using a software like e. g. EAGLE [2]. In the second step the designed PCB is imported into AEDT and prepared for simulations. In the third step a co-simulation between ANSYS HFSS Design and ANSYS Circuit Design is used to combine the results of field simulations with S-parameters of the used components. In this section the two last steps will be described.

A PCB layout can be imported to an AEDT model as a Drawing Exchange Format (DXF) file. However, in DXF, information about the thickness and material of each layer as well as via dimensions are lost. Therefore the relevant material data must be manually set. Also irrelevant layers like silkscreen can be removed. The correct recreation of vias is critical as only their positions are kept in the DXF format. In designs with only few vias this may be done manually. However, for designs with many vias, it would be too time consuming and error prone so that we decided to develop a MATLAB [3] script for automatic placement of all vias. The resulting simulation procedure is shown in Fig. 1. From the layout software two files in Gerber format (GBR) that contain coordinates and diameters of all vias can be exported. These files are used by the MATLAB script to create a script in

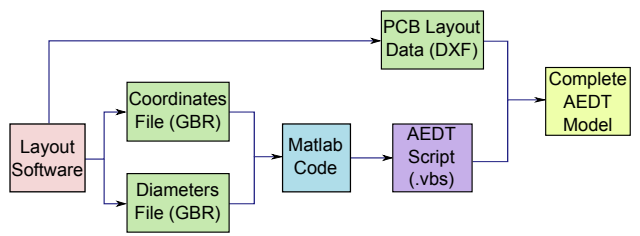

Fig. 1: Flow-chart of the simulation procedure.

Visual Basic Scripting Edition (VBScript or .vbs) that can be read by AEDT. The complete AEDT model including all vias can be then obtained by running the VBSctipt on the DXF file containing the PCB layout.

In order to analyze the PCB, ports must be defined in the AEDT model at the input and output, but also where components will be connected to the structure. The ports are created for the first simulation analyzing the PCB structure alone. During the co-simulation, they are used to connect the $\mathrm{PCB}$ with the component models. To perform the co-simulation, S-parameter files (touchstone format) of the components are required. They are usually provided by the manufacturers. Models of simple lumped elements as resistors and capacitors can also be found in the component library of AEDT.

\section{ANALYZED RF CIRCUITS}

To validate the simulation technique, two circuits were simulated. The substrate was a two-layer FR4 board, with a height of $1 \mathrm{~mm}$.

\section{A. Low-Noise Amplifier Chain}

First, a circuit with two Hittite HMC-320 low-noise amplifiers (LNAs) [4] for $5-6 \mathrm{GHz}$ was analyzed. The assembled PCB is shown in Fig. 2. The two LNAs are connected with coupling capacitors between them and the SMA connectors. Bias resistors and blocking capacitors are necessary for the $5 \mathrm{~V}$ power supply. According to data sheet, the expected gain for this chain is $22 \mathrm{~dB}$, input return loss should be $<-10 \mathrm{~dB}$. Fig. 3 shows the resulting AEDT model for co-simulation with the connections between PCB and components. The peripheral components for the LNAs are neglected in the simulation because, naturally, no S-parameters are available for the supply ports of the amplifiers. 


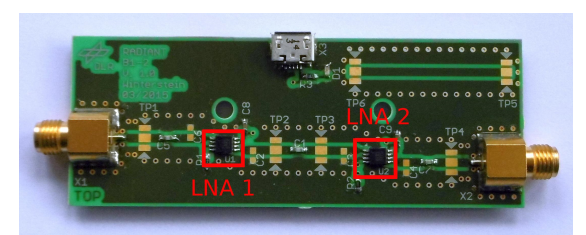

Fig. 2: PCB with SMA connectors, coupling capacitors, two LNAs, and peripheral components.

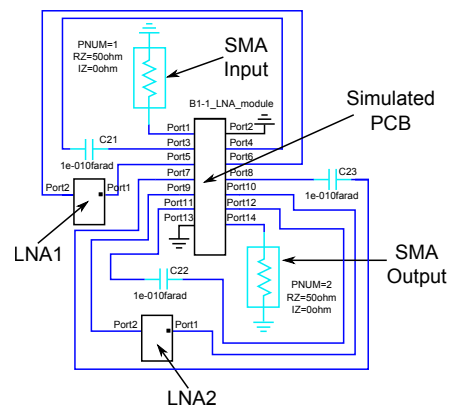

Fig. 3: Designed circuit for the LNA co-simulation.

\section{B. Medium Power Amplifier}

The same process used to simulate the LNA circuit was adopted to simulate a PCB with a Mini-Circuits ERA-1SM+ medium power amplifier (MPA) [5]. The chosen MPA is a broadband amplifier operating from 0 to $8 \mathrm{GHz}$, however the results are shown between $20-200 \mathrm{MHz}$ due the required central frequency of operation $100 \mathrm{MHz}$. It is composed of $\mathrm{AC}$ and DC as feeding system that was not included in the simulation as explained before. The RF circuit is composed of following components: MPA is connected with coupling capacitors between them and the SMA connectors, bias resistors and blocking capacitors are necessary for the $12 \mathrm{~V}$ power supply and RF choke to keep the RF signal in the RF circuit. According to data sheet, the expected gain for this chain and frequency is around $12 \mathrm{~dB}$, input return loss should be $<-10 \mathrm{~dB}$.

\section{Comparison with Measured Results}

The simulated and measured S-parameters of the LNA and MPA circuits are shown in Fig. 4 and Fig. 5 respectively. It can be seen that there is generally good agreement between the results. Especially the forward gain $\left(S_{21}\right)$ is of interest and it agrees quite well with the measurements and also the expected values. For the LNAs, we get a divergence of $<4 \mathrm{~dB}$, for the MPA, it is $<1 \mathrm{~dB}$. The input matching $\left(S_{11}\right)$ also shows very good agreement with measurements being slightly better for both PCBs. The discrepancy between simulated and measured results for the backward gain and output matching is higher but still acceptable.

There are three main reasons for the discrepancies. First, the simulations were performed with S-parameters supplied by the manufacturer. The measurement setup from which these values were obtained is not known and was certainly not

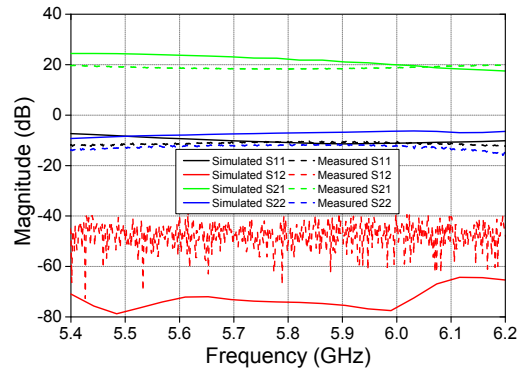

Fig. 4: S-parameters for the LNA circuit.

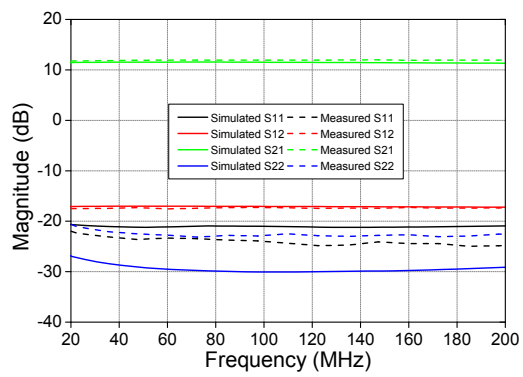

Fig. 5: S-parameters for the MPA circuit.

the same as used in this work. A different choice of PCB material, thickness, layer stack-up or footprint design and the use of connectors will lead to variations in the results. The use of coupling capacitors influences return loss. Second, as described in II, the simulation uses lumped ports for the interconnection of the components. This is an idealized assumption for the soldered connections in the real circuits. Third, FR4 behaves anisotropically at high frequencies but in the simulations a constant permittivity of 4.4 was assumed. Additionally the influence of solder mask was neglected as the material parameters were not known. The impact of these shortcomings increases for higher frequencies but is still tolerable as can be seen in the results.

\section{CONClusion}

In this paper we have proposed a technique to predict the performance of RF amplifier circuits using ANSYS simulation software. Results are obtained from PCB design data and component S-parameters which are available from suppliers. We have shown necessary steps to setup the simulation. The concept has been validated using two example circuits in different frequency bands. Very good agreement between simulation and measured results has been observed.

\section{REFERENCES}

[1] ANSYS Corp., ANSYS Electronic Desktop - AED, version 16., 2015.

[2] CadSoft Corp., Easily Applicable Graphical Layout Editor - EAGLE, version 7.2.0., 2013.

[3] MathWorks, Inc., Matrix Laboratory - MATLAB, version 7.12.0.635 (R2011a), 2011

[4] HMC320MS8G / 320MS8GE, v03.0308 ed., Hittite Microwave Corporation, Chelmsford, MA 01824. [Online]. Available: www.analog.com

[5] ERA-ISM+, Mini-Circuits Corporation, Brooklyn, NY 11235-0003. [Online]. Available: www.minicircuits.com 